\title{
Stackelberg Strategies in Linear-Quadratic Stochastic Differential Games ${ }^{1}$
}

\author{
A. BAGCHI ${ }^{2}$ AND T. BAŞAR ${ }^{3}$ \\ Communicated by Y. C. Ho
}

\begin{abstract}
This paper obtains the Stackelberg solution to a class of two-player stochastic differential games described by linear state dynamics and quadratic objective functionals. The information structure of the problem is such that the players make independent noisy measurements of the initial state and are permitted to utilize only this information in constructing their controls. Furthermore, by the very nature of the Stackelberg solution concept, one of the players is assumed to know, in advance, the strategy of the other player (the leader). For this class of problems, we first establish existence and uniqueness of the Stackelberg solution and then relate the derivation of the leader's Stackelberg solution to the optimal solution of a nonstandard stochastic control problem. This stochastic control problem is solved in a more general context, and its solution is utilized in constructing the Stackelberg strategy of the leader. For the special case Gaussian statistics, it is shown that this optimal strategy is affine in observation of the leader. The paper also discusses numerical aspects of the Stackelberg solution under general statistics and develops algorithms which converge to the unique Stackelberg solution.
\end{abstract}

Key Words. Stochastic differential games, Stackelberg solution, linear-quadratic games, nonzero-sum games.

\section{Introduction}

The Stackelberg solution concept, originally introduced by $\mathrm{H}$. Von Stackelberg in the context of static economic equilibrium (Ref. 1), has entered the control literature through the works of Chen, Cruz, and Simaan

\footnotetext{
${ }^{1}$ This work was performed while the second author was on sabbatical leave at the Department of Applied Mathematics, Twente University of Technology, Enschede, Holland.

${ }^{2}$ Senior Lecturer, Department of Applied Mathematics, Twente University of Technology, Enschede, Holland.

${ }^{3}$ Senior Research Scientist, Applied Mathematics Division, Marmara Scientific and Industrial Research Institute, Gebze, Kocaeli, Turkey. Presently, Associate Professor, Coordinated Science Laboratory, University of Illinois, Urbana, Illinois.
} 
(Refs. 2-4), and has found applications in hierarchical control and differential game problems (Refs. 5-7). Our interest in this paper lies in solving a class of stochastic nonzero-sum differential games with (nonnested) static information under the Stackelberg solution concept.

The first result in the literature on a stochastic Stackelberg problem can be found in Ref. 8, where the authors obtain the feedback Stackelberg solution of a two-player linear-quadratic-Gaussian (LQG) nonzero-sum discrete-time dynamic game problem under a nested information pattern. With regard to nonnested information patterns, Refs. 5 and 9 solve a class of static stochastic Stackelberg game problems when the underlying statistics of the random variables are not necessarily Gaussian. Some of these results are then utilized in Ref. 10 to obtain the feedback Stackelberg solution of the LQG problem of Ref. 8 under a one-step-delay information sharing pattern.

In this paper, we consider the two-player continuous-time LQ nonzerosum differential game under general statistics and under a (nonnested) static information for both players. We establish existence and uniqueness of the Stackelberg solution, and determine it as the solution of an integral equation.

The special case of Gaussian statistics is also treated in the paper, and it is shown that the Stackelberg strategies are then affine in the available static information. Finally, the paper includes a discussion on the computational aspects of the solution.

In Section 2, we formulate our problem and prove existence and uniqueness of Stackelberg strategies. Furthermore, we outline the underlying idea of our method of solution. This leads to a nonstandard stochastic control problem where the state equation contains an extra term involving conditional expectation of the control term, and where some of the components of the state vector have their initial conditions while others have their terminal conditions specified. In Section 3, we solve a general stochastic control problem of this type; and, in Section 4, we use these results to obtain the Stackelberg strategies. The special case of Gaussian statistics is treated in Section 5, while numerical aspects are discussed in Section 6. The paper ends with a conclusions section.

\section{Problem Formulation and Existence Consideration}

Let $\left\{x_{t}, t \geqslant t_{0}\right\}$ denote an $n$-dimensional stochastic process satisfying the Itô stochastic differential equation

$$
\begin{aligned}
& d x_{t}=\left[A(t) x_{t}+B^{1}(t) u_{t}^{1}+B^{2}(t) u_{t}^{2}\right] d t+F(t) d W_{t}, \\
& t \geqslant t_{0}, \quad x_{t_{0}}=x_{0},
\end{aligned}
$$


and whose sample paths are continuous. Here, $x_{0}$ is a random vector with known statistics, such that $E\left|x_{0}\right|<\infty$, and $\left\{W_{t}, t \geqslant t_{0}\right\}$ is an $n$-dimensional standard Brownian motion. $A(\cdot), B^{1}(\cdot), B^{2}(\cdot), F(\cdot)$ are appropriate dimensional matrices with continuous entries on $\left[t_{0}, t_{f}\right] .\left\{u_{t}^{1}, t \geqslant t_{0}\right\}$ and $\left\{u_{t}^{2}\right.$, $\left.t \geqslant t_{0}\right\}$ are, respectively, $r_{1}$-dimensional and $r_{2}$-dimensional stochastic processes denoting the controls of decision makers DM1 and DM2, respectively.

The decision makers make independent noisy measurements of the initial state $x_{0}$, which we denote by $y^{i}, i=1,2$. We assume that the conditional joint distribution of $\left(y^{1}, y^{2}\right)$, given $x_{0}$, is a priori known. The information available to each DM, therefore, is static in nature. Let $H^{i}, i=1,2$, denote the class of second-order stochastic processes defined on $\left[t_{0}, t_{f}\right]$ which are $y^{i}$-measurable.

The decision law (or strategy) $\gamma^{i}$ of DM $i$ is a real Borel-measurable mapping

$$
\left[t_{0}, t_{f}\right] \times \mathbb{R}^{m_{i}} \rightarrow \mathbb{R}^{r_{i}},
$$

where $m_{i}$ denotes the dimension of $y^{i}, i=1,2$. Denoting the space of all Borel-measurable functions

$$
f:\left[t_{0}, t_{f}\right] \times \mathbb{R}^{m_{i}} \rightarrow \mathbb{R}^{r_{i}},
$$

with the further restriction that

$$
E \int_{t_{0}}^{t_{f}}\left\|f\left(t, y^{i}\right)\right\|^{2} d t<\infty
$$

by $\Gamma^{i}$, we require each permissible $\gamma^{i}$ to be in $\Gamma^{i}$. Clearly, $\gamma^{i}\left(\cdot, y^{i}\right)$ is in $H^{i}$. It should be noted that, for each pair of elements in $H^{1} \times H^{2}$, the stochastic differential equation (1) admits a unique solution whose sample paths are continuous (Ref. 11).

For each $\left\{\gamma^{1} \in \Gamma^{1}, \gamma^{2} \in \Gamma^{2}\right\}$, we now define the quadratic objective functionals of DM1 and DM2, respectively, as

$$
\begin{aligned}
& J_{1}\left(\gamma^{1}, \gamma^{2}\right)= E\left\{x_{t_{f}^{\prime}}^{\prime} Q_{1 f} x_{t_{f}}+\int_{t_{0}}^{t_{f}}\left(x_{t}^{\prime} Q_{1}(t) x_{t}+u_{t}^{1^{\prime}} u_{t}^{1}\right.\right. \\
&\left.\left.+u_{t}^{2^{\prime}} R(t) u_{t}^{2}\right) d t \mid u_{t}^{i}=\gamma^{i}\left(t, y^{i}\right), i=1,2\right\}, \\
& J_{2}\left(\gamma^{1}, \gamma^{2}\right)= E\left\{x_{t_{f}^{\prime}}^{\prime} Q_{2 f} x_{t_{f}}+\int_{t_{0}}^{t_{f}}\left(x_{t}^{\prime} Q_{2}(t) x_{t}+u_{t}^{2^{\prime}} u_{t}^{2}\right) d t\right\} \\
&\left.u_{t}^{i}=\gamma^{i}\left(t, y^{i}\right), i=1,2\right\},
\end{aligned}
$$

with prime denoting transpose of a matrix, where

$$
Q_{1 f} \geqslant 0, \quad Q_{2 f} \geqslant 0, \quad Q_{1}(\cdot) \geqslant 0, \quad Q_{2}(\cdot) \geqslant 0, \quad R(\cdot) \geqslant 0,
$$


where the last three matrix-valued functions have continuous entries on $\left[t_{0}, t_{f}\right]$ and the expectation operation is taken over the underlying statistics.

We note at this point that, because of the hierarchical nature of the solution concept to be introduced below, which allows DM2 to have access to the strategy of DM1, Eqs. (2) and (3) define the most general quadratic convex functionals for the decision makers when the cross terms involving the two strategies do not explicitly appear.

To introduce the hierarchical (Stackelberg) solution concept, let us first note that, for each $\bar{\gamma}^{1} \in \Gamma^{1}$, there exists a unique $\bar{\gamma}^{2} \in \Gamma^{2}$ that minimizes $J_{2}\left(\bar{\gamma}^{1}, \gamma^{2}\right)$ over $\Gamma^{2}$. This, then, implies the existence of a unique map $T: \Gamma^{1} \rightarrow \Gamma^{2}$ such that

$$
J_{2}\left(\gamma^{1}, T \gamma^{1}\right) \leqslant J_{2}\left(\gamma^{1}, \gamma^{2}\right)
$$

for all $\gamma^{2} \in \Gamma^{2}$ and for every $\gamma^{1} \in \Gamma^{1}$. Now, consider the minimization of the functional $J_{1}\left(\gamma^{1}, T \gamma^{1}\right)$ over $\Gamma^{1}$, and let $\gamma^{1^{10}} \in \Gamma^{1}$ denote one minimizing solution; that is,

$$
J_{1}\left(\gamma^{1^{0}}, T \gamma^{10}\right) \leqslant J_{1}\left(\gamma^{1}, T \gamma^{1}\right), \quad \forall \gamma^{1} \in \Gamma^{1} .
$$

Then, we say that the pair $\left(\gamma^{10}, \gamma^{20}=T \gamma^{10}\right)$ provides a Stackelberg solution for the game problem under consideration, with DM1 as the leader. Our objective in this paper is to study the existence, uniqueness, and (most importantly) characterization of the Stackelberg solution of the nonzerosum linear-quadratic stochastic differential game formulated above.

The existence and uniqueness questions may be answered most readily by converting the problem into an equivalent hierarchical static game problem in Hilbert space. Barring the stochastic aspect, our approach is identical to that in Ref. 3. We introduce an inner product $\langle\cdot, \cdot\rangle_{i}$ on $H^{i}$ through the relation

$$
\langle u, v\rangle_{i}=E\left\{\int_{t_{0}}^{t_{f}} u_{t}(\omega)^{\prime} v_{t}(\omega) d t\right\}
$$

for each pair $\left\{u \in H^{i}, v \in H^{i}\right\}, \omega \in \Omega$, with $(\Omega, \mathscr{B}, \mathscr{P})$ denoting the underlying probability space. With these inner products, $H^{i}, i=1,2$, become Hilbert spaces. Let $L_{2 f}\left(\left[t_{0}, t_{f}\right] \times \Omega\right)$ denote the completion of the space of continuous functions from $\left[t_{0}, t_{f}\right] \times \Omega$ into $\mathbb{R}^{n}$, under the inner product

$$
\langle x, z\rangle_{f}=E\left\{x_{t_{f}}(\omega)^{\prime} z_{t_{f}}(\omega)+\int_{t_{0}}^{t_{f}} x_{t}(\omega)^{\prime} z_{t}(\omega) d t\right\} .
$$

Further, let $\mathscr{Q}_{i}, i=1,2$, be the operators mapping $L_{2 f}\left(\left[t_{0}, t_{f}\right] \times \Omega\right)$ into itself, 
defined by

$$
\left(\mathscr{Q}_{i} x\right)_{t}(\omega)= \begin{cases}Q_{i}(t) x_{t}(\omega), & t_{0} \leqslant t<t_{f}, \\ Q_{i f} x_{t_{f}}(\omega), & t=t_{f} .\end{cases}
$$

Define Volterra operators

$$
\mathscr{L}_{i}: H^{i} \rightarrow L_{2 f}\left(\left[t_{0}, t_{f}\right] \times \Omega\right), \quad i=1,2,
$$

by

$$
\left(\mathscr{L}_{i} u\right)_{t}(\omega)=\int_{t_{0}}^{t} \Phi(t, s) B^{i}(s) u_{s}(\omega) d s
$$

where $\Phi(t, s)$ is the state transition matrix function satisfying

$$
d \Phi(t, s) / d t=A(t) \Phi(t, s), \quad \Phi(s, s)=I .
$$

Further, let

$$
r \in L_{2 f}\left(\left[t_{0}, t_{f}\right] \times \Omega\right)
$$

be defined by

$$
r_{i}(\omega)=\Phi\left(t, t_{0}\right) x_{0}(\omega)+\int_{t_{0}}^{t} \Phi(t, s) F(s) d W_{s}(\omega) .
$$

The solution of (1) can then be expressed as

$$
x=r+\mathscr{L}_{1} \gamma^{1}\left(\cdot, y^{1}\right)+\mathscr{L}_{2} \gamma^{2}\left(\cdot, y^{2}\right) .
$$

Then, the quadratic objective functionals of DM1 and DM2 respectively become

$$
\begin{aligned}
J_{1}\left(\gamma^{1}, \gamma^{2}\right)= & \left\langle\mathscr{Q}_{1}\left(\mathscr{L}_{1} \gamma^{1}\left(\cdot, y^{1}\right)+\mathscr{L}_{2} \gamma^{2}\left(\cdot, y^{2}\right)+r\right), \mathscr{L}_{1} \gamma^{1}\left(\cdot, y^{1}\right)\right. \\
& \left.+\mathscr{L}_{2} \gamma^{2}\left(\cdot, y^{2}\right)+r\right\rangle_{f}+\left\langle\gamma^{1}\left(\cdot, y^{1}\right), \gamma^{1}\left(\cdot, y^{1}\right)\right\rangle_{1} \\
& +\left\langle\gamma^{2}\left(\cdot, y^{2}\right), R \gamma^{2}\left(\cdot, y^{2}\right)\right\rangle_{2}, \\
J_{2}\left(\gamma^{1}, \gamma^{2}\right)= & \left\langle\mathscr{Q}_{2}\left(\mathscr{L}_{1} \gamma^{1}\left(\cdot, y^{1}\right)+\mathscr{L}_{2} \gamma^{2}\left(\cdot, y^{2}\right)+r\right), \mathscr{L}_{1} \gamma^{1}\left(\cdot, y^{1}\right)\right. \\
& \left.+\mathscr{L}_{2} \gamma^{2}\left(\cdot, y^{2}\right)+r\right\rangle_{f}+\left\langle\gamma^{2}\left(\cdot, y^{2}\right), \gamma^{2}\left(\cdot, y^{2}\right)\right\rangle_{2},
\end{aligned}
$$

where

$$
R: H^{2} \rightarrow H^{2}
$$

is defined by

$$
(R u)_{t}(\omega)=R(t) u_{t}(\omega), \quad t_{0} \leqslant t \leqslant t_{f} .
$$

For fixed $\gamma^{1} \in \Gamma^{1}$, minimization of $J_{2}\left(\gamma^{1}, \gamma^{2}\right)$ is equivalent to the control 
problem of minimizing

$$
J_{2}\left(u^{2}\right)=\left\langle\mathscr{Q}_{2}\left(\mathscr{L}_{1} \gamma^{1}\left(\cdot, y^{1}\right)+\mathscr{L}_{2} u^{2}+r\right), \mathscr{L}_{1} \gamma^{1}\left(\cdot, y^{1}\right)+\mathscr{L}_{2} u^{2}+r\right\rangle_{f}+\left\langle u^{2}, u^{2}\right\rangle_{2},
$$

for $u^{2} \in H^{2}$. Now, $I+\mathscr{L}_{2}^{*} \mathscr{Q}_{2} \mathscr{L}_{2}$ being strongly positive and having a bounded inverse $\left(\mathscr{L}_{2}^{*}\right.$ denotes the adjoint of $\mathscr{L}_{2}$ in $\left.H^{2}\right), J_{2}\left(u^{2}\right)$ has the unique minimum

$$
u^{2}=-\left(I+\mathscr{L}_{2}^{*} \mathscr{Q}_{2} \mathscr{L}_{2}\right)^{-1} \mathscr{L}_{2}^{*} \mathscr{Q}_{2}\left(\mathscr{L}_{1} \gamma^{1}\left(\cdot, y^{1}\right)+r\right) .
$$

Substituting this expression for $\gamma^{2}\left(\cdot, y^{2}\right)$ in (9) and rearranging terms, we get, in terms of the notation discussed above,

$$
\begin{aligned}
J_{1}\left(\gamma^{1}, T \gamma^{1}\right)= & \left\langle\gamma^{1}\left(\cdot, y^{1}\right),(I+\overline{\mathscr{R}}) \gamma^{1}\left(\cdot, y^{1}\right)\right\rangle_{1}+2\left\langle\gamma^{1}\left(\cdot, y^{1}\right), \tilde{\mathscr{L}}_{1}^{*} \mathscr{Q}_{1} \tilde{r}\right. \\
& \left.+\mathscr{L}_{1}^{*} \mathscr{Q}_{2} \mathscr{L}_{2}\left(I+\mathscr{L}_{2}^{*} \mathscr{Q}_{2} \mathscr{L}_{2}\right)^{-1} R\left(I+\mathscr{L}_{2}^{*} \mathscr{Q}_{2} \mathscr{L}_{2}\right)^{-1} \mathscr{L}_{2}^{*} \mathscr{Q}_{2} r\right\rangle_{1}+J_{10},
\end{aligned}
$$

where $J_{10}$ is independent of $\gamma^{1}$,

$$
\begin{aligned}
\overline{\mathscr{R}} & =\tilde{\mathscr{L}}_{1}^{*} \mathscr{Q}_{1} \tilde{\mathscr{L}}_{1}+\mathscr{L}_{1}^{*} \mathscr{Q}_{2} \mathscr{L}_{2}\left(I+\mathscr{L}_{2}^{*} \mathscr{Q}_{2} \mathscr{L}_{2}\right)^{-1} R\left(I+\mathscr{L}_{2}^{*} \mathscr{Q}_{2} \mathscr{L}_{2}\right)^{-1} \mathscr{L}_{2}^{*} \mathscr{Q}_{2} \mathscr{L}_{1}, \\
\tilde{\mathscr{L}}_{1} & =\mathscr{L}_{1}-\mathscr{L}_{2}\left(I+\mathscr{L}_{2}^{*} \mathscr{Q}_{2} \mathscr{L}_{2}\right)^{-1} \mathscr{L}_{2}^{*} \mathscr{Q}_{2} \mathscr{L}_{1}, \\
\tilde{r} & =\left(I-\mathscr{L}_{2}\left(I+\mathscr{L}_{2}^{*} \mathscr{Q}_{2} \mathscr{L}_{2}\right)^{-1} \mathscr{L}_{2}^{*} \mathscr{Q}_{2}\right) r,
\end{aligned}
$$

and $\tilde{\mathscr{L}}_{1}^{*}$ is the adjoint of $\tilde{\mathscr{L}}_{1}$ under the inner product $(5 \mathrm{~b})$. Again, $I+\overline{\mathscr{R}}$ being strongly positive and having a bounded inverse, taking variations in $\Gamma^{1}$, we can readily show that there exists a unique $\gamma^{10}$ for which $J_{1}\left(\gamma^{1}, T \gamma^{1}\right)$ has a minimum. Then, this guarantees existence of a unique Stackelberg solution for the nonzero-sum stochastic differential game above.

Let us now outline our method of solution. For each $\gamma^{1} \in \Gamma_{1}$, the control $u^{2} \in H^{2}$ that minimizes $J_{2}\left(u^{2}\right)$ is given by (see Ref. 12 for details):

$$
u_{t}^{2}=-B^{2}(t)^{\prime}\left\{S^{2}(t) E\left[x_{t} \mid y^{2}\right]+k_{t}^{2}\right\},
$$

where

$$
\begin{array}{r}
\dot{S}^{2}(t)=-A(t)^{\prime} S^{2}(t)-S^{2}(t) A(t)-Q_{2}(t)+S^{2}(t) B^{2}(t) B^{2}(t)^{\prime} S^{2}(t), \\
S^{2}\left(t_{f}\right)=Q_{2 f}, \\
\dot{k}_{t}^{2}=-\left(A(t)^{\prime}-S^{2}(t) B^{2}(t) B^{2}(t)^{\prime}\right) k_{t}^{2}-S^{2}(t) B^{1}(t) E\left[\gamma^{1}\left(t, y^{1}\right) \mid y^{2}\right], \\
k_{t_{f}}^{2}=0 ;
\end{array}
$$

and let us denote $E\left[x_{t} \mid y^{2}\right]$ by $\hat{x}_{t}^{2}$. Then,

$$
\begin{aligned}
d \hat{x}_{t}^{2}= & \left(A(t)-B^{2}(t) B^{2}(t)^{\prime} S^{2}(t)\right) \hat{x}_{t}^{2} d t-B^{2}(t) B^{2}(t)^{\prime} k_{t}^{2} d t \\
& +B^{1}(t) E\left[\gamma^{1}\left(t, y^{1}\right) \mid y^{2}\right] d t
\end{aligned}
$$




$$
\hat{x}_{t_{0}}^{2}=E\left[x_{t_{0}} \mid y^{2}\right]=E\left[x_{0} \mid y^{2}\right]
$$

Furthermore, if

$$
e_{t}=x_{t}-\hat{x}_{t}^{2}
$$

then

$$
\begin{aligned}
d e_{t}= & A(t) e_{t} d t+B^{1}(t) \gamma^{1}\left(t, y^{1}\right) d t \\
& -B^{1}(t) E\left[\gamma^{1}\left(t, y^{1}\right) \mid y^{2}\right] d t+F(t) d W_{t}, \\
e_{t_{0}}= & x_{0}-E\left[x_{0} \mid y^{2}\right] .
\end{aligned}
$$

In terms of these newly introduced variables $\hat{x}_{t}^{2}, e_{t}, k_{t}^{2}$, we can express $J_{1}$ as follows:

$$
\begin{aligned}
J_{1}\left(\gamma^{1}, T \gamma^{1}\right)= & \hat{x}_{t_{f}}^{2^{\prime}} Q_{1 f} \hat{x}_{t_{f}}^{2}+e_{t_{f}}^{\prime} Q_{1 f} e_{t_{f}}+\int_{t_{0}}^{t_{f}}\left(\hat{x}_{t}^{2} Q_{1}(t) \hat{x}_{t}^{2}+e_{t}^{t} Q_{1}(t) e_{t}\right. \\
& +\left\{\hat{x}_{t}^{2} S^{2}(t)+k_{t}^{2}\right\} B^{2}(t) R(t) B^{2}(t)^{\prime}\left\{S^{2}(t) \hat{x}_{t}^{2}+k_{t}^{2}\right\} \\
& \left.+\gamma^{1}\left(t, y^{1}\right)^{\prime} \gamma^{1}\left(t, y^{1}\right)\right) d t
\end{aligned}
$$

Therefore, the leader is faced with a nonstandard stochastic optimization problem, the general formulation and solution of which is discussed in the next section.

\section{Digression: Nonstandard Stochastic Control Problem}

Let us consider a stochastic system whose state evolves according to the following stochastic differential equation:

$$
d x_{t}=\left\{A x_{t}+B \gamma(t, y)+C E[\gamma(t, y) \mid z]\right\} d t+d w_{t},
$$

where $x_{t}$ is an $n$-dimensional stochastic process; $y$ and $z$ are $p$-dimensional and $q$-dimensional random vectors, respectively; $\gamma:\left[t_{0}, t_{f}\right] \times \mathbb{R}^{p} \rightarrow \mathbb{R}^{m}$, and all admissible $\gamma$ belong to $\Gamma$, with $\Gamma$ being a linear vector space; $w_{t}$ is $n$-dimensional Brownian motion, and $A, B, C$ are appropriate dimensional matrices. We are interested in the situation where some components of $x_{t}$ are specified at the initial time $t_{0}$ and the rest are specified at the terminal time $t_{f}$. Let us instead consider a slightly more general set up. Thus, there exist matrices $T_{0}$ and $T_{f}$ such that

$$
T_{0} x_{t_{0}}=\pi, \quad T_{f} x_{t_{f}}=\chi
$$

are given. To ensure that all the components of $x_{t}$ are specified at one of the endpoints, we impose the restriction that

$$
T_{0}^{\prime} T_{0}+T_{f}^{\prime} T_{f}=I \text {. }
$$


Furthermore, in order to avoid some cumbersome expressions in the analysis to follow, we assume that the system matrix is structured so that

$$
T_{f} A T_{0}^{\prime}=0
$$

which is a condition that is fulfilled for the Stackelberg problem under consideration (see Section 4). Finally, we assume that $\Gamma$ is chosen such that, for each $\gamma \in \Gamma$, there exists a unique solution of (18) satisfying the boundary conditions (19a).

Our problem then is to determine $\gamma \in \Gamma$ for which

$$
J(\gamma)=E\left\{x_{t_{f}}^{\prime} Q_{f} x_{t_{f}}+\int_{t_{0}}^{t_{f}}\left[x_{t}^{\prime} Q(t) x_{t}+\gamma(t, y)^{\prime} \gamma(t, y)\right] d t\right\}
$$

attains a minimum, where

$$
Q_{f} \geqslant 0, \quad Q(t) \geqslant 0, \quad \text { for a.e. } t \in\left[t_{0}, t_{f}\right] .
$$

We use standard variational argument to solve this problem. If we perturb $\gamma$ to $\gamma+\epsilon \beta$, suppose that the state $x_{t}$ is perturbed to $x_{t}^{\epsilon}$. Then,

$$
\begin{aligned}
d x_{t}^{\epsilon}= & \left\{A x_{t}^{\epsilon}+B \gamma(t, y)+\epsilon B \beta(t, y)+C E[\gamma(t, y) \mid z]\right. \\
& +\epsilon C E[\beta(t, y) \mid z]\} d t+d w_{t}, \\
& T_{0} x_{t_{0}}^{\epsilon}=\pi, \quad T_{f} x_{t_{f}}^{\epsilon}=\chi .
\end{aligned}
$$

We can clearly decompose $x_{t}^{\epsilon}$ as $x_{t}+\epsilon \eta_{t}$ where

$$
\begin{gathered}
\dot{\eta}_{t}=A \eta_{t}+B \beta(t, y)+C E[\beta(t, y) \mid z], \\
T_{0} \eta_{t_{0}}=0, \quad T_{f} \eta_{t_{f}}=0 .
\end{gathered}
$$

Taking first variation in $J$, a necessary condition for minimum is that

$$
\delta_{1} J(\gamma ; \beta)=E\left\{\eta_{t_{f}}^{\prime} Q_{f} x_{t_{f}}+\int_{t_{0}}^{t_{f}}\left[\eta_{t}^{\prime} Q(t) x_{t}+\beta(t, y)^{\prime} \gamma(t, y)\right] d t\right\}=0, \quad \forall \beta \in \Gamma .
$$

Since the second variation is positive, the above condition is also sufficient.

Define $\eta_{t}^{1}$ and $\eta_{t}^{2}$ by

$$
\begin{array}{lll}
\dot{\eta}_{t}^{1}=A \eta_{t}^{1}+B \beta(t, y), & T_{0} \eta_{t_{0}}^{1}=0, & T_{f} \eta_{t_{f}}^{1}=0, \\
\dot{\eta}_{t}^{2}=A \eta_{t}^{2}+C \beta(t, y), & T_{0} \eta_{t_{0}}^{2}=0, & T_{f} \eta_{t_{f}}^{2}=0 .
\end{array}
$$

Then,

$$
\eta_{t}=\eta_{t}^{1}+E\left[\eta_{t}^{2} \mid z\right]
$$


and the necessary and sufficient condition for minimum may be reexpressed as

$$
\begin{aligned}
\delta_{1} J(\gamma ; \beta)= & E\left\{\eta_{t_{f}}^{1^{\prime}} Q_{f} E\left[x_{t_{f}} \mid y\right]+E\left[\eta_{t_{f}}^{2^{\prime}} \mid z\right] Q_{f} E\left[x_{t_{f}} \mid z\right]\right. \\
& +\int_{t_{0}}^{t_{f}}\left[\eta_{t}^{1^{\prime}} Q(t) E\left[x_{f} \mid y\right]+E\left[\eta_{t}^{2^{\prime}} \mid z\right] Q(t) E\left[x_{t} \mid z\right]\right. \\
& \left.\left.+\beta(t, y)^{\prime} \gamma(t, y)\right] d t\right\}=E\left\{\eta_{t_{f}}^{1^{\prime}} Q_{f} E\left[x_{t_{f}} \mid y\right]+\eta_{t_{f}}^{2^{\prime}} Q_{f} E\left[E\left[x_{t_{f}} \mid z\right] \mid y\right]\right. \\
& +\int_{t_{0}}^{t_{f}}\left[\eta_{t}^{1^{\prime}} Q(t) E\left[x_{t} \mid y\right]+\eta_{t}^{2^{\prime}} Q(t) E\left[E\left[x_{t} \mid z\right] \mid y\right]\right. \\
& \left.\left.+\beta(t, y)^{\prime} \gamma(t, y)\right] d t\right\}=0, \quad \forall \beta \in \Gamma .
\end{aligned}
$$

Let

$$
\hat{x}_{t}^{y}=E\left[x_{t} \mid y\right] \text { and } \hat{x}_{t}^{y}=E\left[E\left[x_{t} \mid z\right] \mid y\right] \text {. }
$$

Then,

$$
\begin{aligned}
\dot{\hat{x}}_{t}^{y} & =A \hat{x}_{t}^{y}+B \gamma(t, y)+C E[E[\gamma(t, y) \mid z] \mid y], \\
T_{0} \hat{x}_{t_{0}}^{y} & =E[\pi \mid y], \quad T_{f} \hat{x}_{t_{f}}^{y}=E[\chi \mid y], \\
\dot{\dot{\hat{x}}_{t}^{y}} & =A \hat{\hat{x}}_{t}^{y}+(B+C) E[E[\gamma(t, y) \mid z] \mid y], \\
T_{0} \hat{\hat{x}}_{t_{0}}^{y} & =E[E[\pi \mid z] \mid y], \quad T_{f} \hat{\hat{x}}_{t_{f}}^{y}=E[E[\chi \mid z] \mid y],
\end{aligned}
$$

and

$$
\begin{aligned}
\delta_{1} J(\gamma ; \beta)= & E\left\{\eta_{t_{f}}^{1^{\prime}} Q_{f} \hat{x}_{t_{f}}^{y}+\eta_{t_{f}^{\prime}}^{2^{\prime}} Q_{f} \hat{\hat{x}}_{t_{f}}^{y}+\int_{t_{0}}^{t_{f}}\left[\eta_{t}^{1^{\prime}} Q(t) \hat{x}_{t}^{y}\right.\right. \\
& \left.\left.+\eta_{t}^{2^{\prime}} Q(t) \hat{\hat{x}}_{t}^{y}+\beta(t, y)^{\prime} \gamma(t, y)\right] d t\right\}=0, \quad \forall \beta \in \Gamma .
\end{aligned}
$$

Note now that, under $(19 \mathrm{c}), T_{f} \eta_{t}^{1}$ and $T_{f} \eta_{t}^{2}$ satisfy, respectively,

$$
\begin{array}{ll}
T_{f} \dot{\eta}_{t}^{1}=T_{f} A T_{f}^{\prime} T_{f} \eta_{t}^{1}+T_{f} B \beta(t, y), & T_{f} \eta_{t_{f}}^{1}=0, \\
T_{f} \dot{\eta}_{t}^{2}=T_{f} A T_{f}^{\prime} T_{f} \eta_{t}^{2}+T_{f} C \beta(t, y), & T_{f} \eta_{t_{f}}^{2}=0,
\end{array}
$$

so that

$$
\begin{aligned}
& T_{f} \eta_{t}^{1}=-\int_{t}^{t_{f}} \Phi_{f}(t, \sigma) T_{f} B(\sigma) \beta(\sigma, y) d \sigma, \\
& T_{f} \eta_{t}^{2}=-\int_{t}^{t_{f}} \Phi_{f}(t, \sigma) T_{f} C(\sigma) \beta(\sigma, y) d \sigma,
\end{aligned}
$$

where

$$
(d / d t) \Phi_{f}(t, \sigma)=T_{f} A T_{f}^{\prime} \Phi_{f}(t, \sigma), \quad \Phi_{f}(\sigma, \sigma)=I
$$


Since

$$
T_{0}^{\prime} T_{0}+T_{f}^{\prime} T_{f}=I,
$$

(21a) and (21b) may be written as

$$
\begin{aligned}
& \dot{\eta}_{t}^{1}=A\left(T_{0}^{\prime} T_{0}+T_{f}^{t} T_{f}\right) \eta_{t}^{1}+B \beta(t, y), \\
& \dot{\eta}_{t}^{2}=A\left(T_{0}^{\prime} T_{0}+T_{f}^{\prime} T_{f}\right) \eta_{t}^{2}+C \beta(t, y) ;
\end{aligned}
$$

and, multiplying further by $T_{0}$, we have

$$
\begin{array}{ll}
T_{0} \dot{\eta}_{t}^{1}=T_{0} A T_{0}^{\prime} T_{0} \eta_{t}^{1}+T_{0} A T_{f}^{\prime} T_{f} \eta_{t}^{1}+T_{0} B \beta(t, y), & T_{0} \eta_{t_{0}}^{1}=0, \\
T_{0} \dot{\eta}_{t}^{2}=T_{0} A T_{0}^{\prime} T_{0} \eta_{t}^{2}+T_{0} A T_{f}^{\prime} T_{f} \eta_{t}^{2}+T_{0} C \beta(t, y), & T_{0} \eta_{t_{0}}^{2}=0,
\end{array}
$$

which imply that

$$
\begin{aligned}
& T_{0} \eta_{t}^{1}=\int_{t_{0}}^{t} \Phi_{0}(t, \sigma)\left[T_{0} B \beta(\sigma, y)+T_{0} A(\sigma) T_{f}^{t} T_{f} \eta_{\sigma}^{1}\right] d \sigma, \\
& T_{0} \eta_{t}^{2}=\int_{t_{0}}^{t} \Phi_{0}(t, \sigma)\left[T_{0} C \beta(\sigma, y)+T_{0} A(\sigma) T_{f}^{\prime} T_{f} \eta_{\sigma}^{2}\right] d \sigma,
\end{aligned}
$$

where

$$
(d / d t) \Phi_{0}(t, \sigma)=T_{0} A T_{0}^{\prime} \Phi_{0}(t, \sigma), \quad \Phi_{0}(\sigma, \sigma)=I .
$$

Therefore,

$$
\begin{aligned}
\eta_{t}^{1}= & T_{0}^{\prime} T_{0} \eta_{t}^{1}+T_{f}^{\prime} T_{f} \eta_{t}^{1} \\
= & T_{0}^{\prime} \int_{t_{0}}^{t} \Phi_{0}(t, \sigma) T_{0} B(\sigma) \beta(\sigma, y) d \sigma-T_{f}^{\prime} \int_{t}^{t_{f}} \Phi_{f}(t, \sigma) T_{f} B(\sigma) \beta(\sigma, y) d \sigma \\
& -T_{0}^{\prime} \int_{t_{0}}^{t} \Phi_{0}(t, \tau) T_{0} A(\tau) T_{f}^{\prime} \int_{\tau}^{t_{f}} \Phi_{f}(\tau, \sigma) T_{f} B(\sigma) \beta(\sigma, y) d \sigma d \tau .
\end{aligned}
$$

Now,

$$
\begin{aligned}
\int_{t}^{t_{f}} & \Phi_{f}(t, \sigma) T_{f} B(\sigma) \beta(\sigma, y) d \sigma=\Phi_{f}\left(t, t_{f}\right)\left[\int_{t_{0}}^{t_{f}} \Phi_{f}\left(t_{f}, \sigma\right) T_{f} B(\sigma) \beta(\sigma, y) d \sigma\right. \\
& \left.-\int_{t_{0}}^{t} \Phi_{f}\left(t_{f}, \sigma\right) T_{f} B(\sigma) \beta(\sigma, y) d \sigma\right]=\int_{t_{0}}^{t_{f}} \Phi_{f}(t, \sigma) T_{f} B(\sigma) \beta(\sigma, y) d \sigma \\
& -\int_{t_{0}}^{t} \Phi_{f}(t, \sigma) T_{f} B(\sigma) \beta(\sigma, y) d \sigma .
\end{aligned}
$$


Furthermore,

$$
\begin{aligned}
- & T_{0}^{\prime} \int_{t_{0}}^{t} \Phi_{0}(t, \tau) T_{0} A(\tau) T_{f}^{\prime} \int_{\sigma}^{t_{f}} \Phi_{f}(\tau, \sigma) T_{f} B(\sigma) \beta(\sigma, y) d \sigma d \tau \\
= & -T_{0}^{\prime} \int_{t_{0}}^{t_{f}} d \sigma \int_{t_{0}}^{t} d \tau \Phi_{0}(t, \tau) T_{0} A(\tau) T_{f}^{\prime} \Phi_{f}(\tau, \sigma) T_{f} B(\sigma) \beta(\sigma, y) d \sigma \\
& +T_{0}^{\prime} \int_{t_{0}}^{t} d \sigma \int_{\sigma}^{t} d \tau \Phi_{0}(t, \tau) T_{0} A(\tau) T_{f}^{\prime} \Phi_{f}(\tau, \sigma) T_{f} B(\sigma) \beta(\sigma, y) d \sigma \\
= & -\int_{t_{0}}^{t_{f}} d \sigma \int_{t_{0}}^{t} d \tau T_{0}^{\prime} \Phi_{0}(t, \tau) T_{0} A(\tau) T_{f}^{\prime} \Phi_{f}(\tau, \sigma) T_{f} B(\sigma) \beta(\sigma, y) d \sigma \\
& +\int_{t_{0}}^{t} d \sigma \int_{\sigma}^{t} d \tau T_{0}^{\prime} \Phi_{0}(t, \tau) T_{0} A(\tau) T_{f}^{\prime} \Phi_{f}(\tau, \sigma) T_{f} B(\sigma) \beta(\sigma, y) d \sigma .
\end{aligned}
$$

Then,

$$
\eta_{t}^{1}=\int_{t_{0}}^{t} P^{1}(t, \sigma) B(\sigma) \beta(\sigma, y) d \sigma-\int_{t_{0}}^{t_{f}} P^{2}(t, \sigma) B(\sigma) \beta(\sigma, y) d \sigma
$$

where

$$
\begin{gathered}
P^{1}(t, \sigma)=T_{0}^{\prime} \Phi_{0}(t, \sigma) T_{0}+T_{f}^{\prime} \Phi_{f}(t, \sigma) T_{f} \\
+\int_{\sigma}^{t}\left[T_{0}^{\prime} \Phi_{0}(t, \tau) T_{0} A(\tau) T_{f}^{\prime} \Phi_{f}(\tau, \sigma) T_{f}\right] d \tau \\
P^{2}(t, \sigma)=T_{f}^{\prime} \Phi_{f}(t, \sigma) T_{f}+\int_{t_{0}}^{t}\left[T_{0}^{\prime} \Phi_{0}(t, \tau) T_{0} A(\tau) T_{f}^{\prime} \Phi_{f}(\tau, \sigma) T_{f}\right] d \tau
\end{gathered}
$$

Analogously,

$$
\eta_{t}^{2}=\int_{t_{0}}^{t} P^{1}(t, \sigma) C(\sigma) \beta(\sigma, y) d \sigma-\int_{t_{0}}^{t_{f}} P^{2}(t, \sigma) C(\sigma) \beta(\sigma, y) d \sigma
$$

Let us now define

$$
\begin{aligned}
P(t, \sigma)= & P^{1}(t, \sigma)-P^{2}(t, \sigma)=T_{0}^{\prime} \Phi_{0}(t, \sigma) T_{0} \\
& -\int_{t_{0}}^{\sigma} d \tau T_{0}^{\prime} \Phi_{0}(t, \tau) T_{0} A(\tau) T_{f}^{\prime} \Phi_{f}(\tau, \sigma) T_{f},
\end{aligned}
$$


and note that

$$
\begin{aligned}
\int_{t_{0}}^{t_{f}} \eta_{t}^{1^{\prime}} Q(t) \hat{x}_{t}^{y} d t=\int_{t_{0}}^{t_{f}} d \sigma \beta^{\prime}(\sigma, y) B^{\prime}(\sigma) \int_{\sigma}^{t_{f}} P^{1}(t, \sigma)^{\prime} Q(t) \hat{x}_{t}^{y} d t \\
\quad-\int_{t_{0}}^{t_{f}} d \sigma \beta^{\prime}(\sigma, y) B^{\prime}(\sigma) \int_{t_{0}}^{t_{f}} P^{2}(t, \sigma)^{\prime} Q(t) \hat{x}_{t}^{y} d t \\
=\int_{t_{0}}^{t_{f}} d \sigma \beta^{\prime}(\sigma, y) B^{\prime}(\sigma)\left[\int_{\sigma}^{t_{f}} P(t, \sigma)^{\prime} Q(t) \hat{x}_{t}^{y} d t-\int_{t_{0}}^{\sigma} P^{2}(t, \sigma)^{\prime} Q(t) \hat{x}_{t}^{y} d t\right] .
\end{aligned}
$$

Analogously,

$$
\begin{gathered}
\int_{t_{0}}^{t_{f}} \eta_{t}^{2^{\prime}} Q(t) \hat{\hat{x}}_{t}^{y} d t=\int_{t_{0}}^{t_{f}} d \sigma \beta^{\prime}(\sigma, y) C^{\prime}(\sigma)\left[\int_{\sigma}^{t_{f}} P(t, \sigma)^{\prime} Q(t) \hat{\hat{x}}_{t}^{y} d t\right. \\
\left.-\int_{t_{0}}^{\sigma} P^{2}(t, \sigma)^{\prime} Q(t) \hat{\hat{x}}_{t}^{y} d t\right] .
\end{gathered}
$$

Utilizing (28a)-(28b) in (24), together with the expressions for $\eta_{t_{f}}^{1}$ and $\eta_{t_{f}}^{2}$, we finally obtain

$$
\delta_{1} J(\gamma ; \beta)=E\left\{\int_{t_{0}}^{t_{f}} \beta(t, y)^{\prime} l(t, y) d t\right\}=0, \quad \forall \beta \in \Gamma,
$$

where

$$
\begin{aligned}
l(t, y)= & \gamma(t, y)+B(t)^{\prime} P\left(t_{f}, t\right)^{\prime} Q_{f} \hat{x}_{t_{f}}^{y}+C(t)^{\prime} P\left(t_{f}, t\right)^{\prime} Q_{f} \hat{\hat{x}}_{t_{f}}^{y} \\
& +B(t)^{\prime} \int_{t}^{t_{f}} P(\sigma, t)^{\prime} Q(\sigma) \hat{x}_{\sigma}^{y} d \sigma+C(t)^{\prime} \int_{t}^{t_{f}} P(\sigma, t)^{\prime} Q(\sigma) \hat{\hat{x}}_{\sigma}^{y} d \sigma \\
& -B(t)^{\prime} \int_{t_{o}}^{t} P^{2}(\sigma, t)^{\prime} Q(t) \hat{x}_{\sigma}^{y} d \sigma-C(t)^{\prime} \int_{t_{0}}^{t} P^{2}(\sigma, t)^{\prime} Q(\sigma) \hat{\hat{x}}_{\sigma}^{y} d \sigma .
\end{aligned}
$$

It now readily follows from (29a) that a necessary and sufficient condition for $\gamma \in \Gamma$ to provide a minimizing solution is that

$$
l(t, y)=0, \quad \forall t \in\left[t_{0}, t_{f}\right], \quad \text { a.e. } \mathscr{P}_{y} .
$$

The following lemma summarizes the result.

Lemma 3.1. A control strategy $\gamma \in \Gamma$ provides a minimizing solution to the stochastic optimum control problem formulated in this section iff it 
satisfies the integral equation

$$
\begin{aligned}
\gamma(t, y)= & -B(t)^{\prime} P\left(t_{f}, t\right)^{\prime} Q_{f} \hat{x}_{t_{f}}^{y}-C(t)^{\prime} P\left(t_{f}, t\right)^{\prime} Q_{f} \hat{\hat{x}}_{f_{f}} \\
& -B(t)^{\prime} \int_{t}^{t_{f}} P(\sigma, t)^{\prime} Q(\sigma) \hat{x}_{\sigma}^{y} d \sigma-C(t)^{\prime} \int_{i}^{t_{f}} P(\sigma, t)^{\prime} Q(\sigma) \hat{x}_{\sigma}^{y} d \sigma \\
& +B(t)^{\prime} \int_{t_{0}}^{t} P^{2}(\sigma, t) Q(\sigma) \hat{x}_{\sigma}^{y} d \sigma+C(t)^{\prime} \int_{t_{0}}^{t} P^{2}(\sigma, t)^{\prime} Q(\sigma) \hat{\hat{x}}_{\sigma}^{y} d \sigma
\end{aligned}
$$

where $\hat{x}_{t}^{y}$ and $\hat{x}_{t}^{y}$ satisfy (23a)-(23b), $P(t, \sigma)$ and $P^{2}(t, \sigma)$ are given by $(27 \mathrm{a})-(27 \mathrm{c})$, and $\Phi_{0}(t, \sigma)$ and $\Phi_{f}(t, \sigma)$ satisfy $(26 \mathrm{c})$ and $(25 \mathrm{c})$, respectively.

\section{Determination of Stackelberg Strategies}

We now go back to our original problem as discussed in Section 2. We have to minimize the cost functional given by Eq. (17), subject to the constraints (16a) through (16c). To this end, we first define the new state vector

$$
\underline{x}_{t}=\left[\begin{array}{c}
\hat{x}_{t}^{2} \\
e_{t} \\
k_{t}^{2}
\end{array}\right]
$$

and the matrices

$$
\begin{aligned}
& A(t)=\left[\begin{array}{ccc}
A(t)-B^{2}(t) B^{2}(t)^{\prime} S^{2}(t) & 0 & -B^{2}(t) B^{2}(t)^{\prime} \\
0 & A(t) & 0 \\
0 & 0 & -\left(A(t)^{\prime}-S^{2}(t) B^{2}(t) B^{2}(t)^{\prime}\right)
\end{array}\right], \\
& B(t)=\left[\begin{array}{c}
0 \\
B^{1}(t) \\
0
\end{array}\right], \quad \underline{C}(t)=\left[\begin{array}{c}
B^{1}(t) \\
-B^{1}(t) \\
-S^{2}(t) B^{1}(t)
\end{array}\right], \quad \underline{F}(t)=\left[\begin{array}{c}
0 \\
F(t) \\
0
\end{array}\right], \\
& Q_{1 f}=\operatorname{diag}\left(Q_{1 f}, Q_{1 f}, 0\right) \text {, } \\
& Q_{1}(t)=\left[\begin{array}{ccc}
Q_{1}(t)+S^{2}(t) B^{2}(t) R(t) B^{2}(t)^{\prime} S^{2}(t) & 0 & S^{2}(t) B^{2}(t) R(t) B^{2}(t)^{\prime} \\
0 & Q_{1}(t) & 0 \\
B^{2}(t) R(t) B^{2}(t)^{\prime} S^{2}(t) & 0 & B^{2}(t) R(t) B^{2}(t)^{\prime}
\end{array}\right] .
\end{aligned}
$$

Then, we have the following minimization problem for player 1: Minimize

$$
J_{1}\left(\gamma^{1}, T \gamma^{1}\right)=\underline{x}_{t_{f}}^{\prime} Q_{1 f} \underline{x}_{t f}+\int_{t_{0}}^{t_{f}}\left[\underline{x}_{t}^{\prime} Q_{1}(t) \underline{x}_{t}+\gamma^{1}\left(t, y^{1}\right)^{\prime} \gamma^{1}\left(t, y^{1}\right)\right] d t
$$


subject to

$$
d \underline{x}_{t}=\left\{\underline{A}(t) \underline{x}_{t}+\underline{B}(t) \gamma^{1}\left(t, y^{1}\right)+\underline{C}(t) E\left[\gamma^{1}\left(t, y^{1}\right) \mid y^{2}\right]\right\} d t+\underline{F}(t) d W_{t},
$$

where $\hat{x}_{t_{0}}^{2}, e_{t_{0}}, k_{t_{f}}^{2}$ are given.

This stochastic optimum control problem can readily be solved by applying the result of Lemma 3.1. To this end, $x_{t}, A, B, C, F$ of Section 3 must be identified with $\underline{x}_{t}, \underline{A}, \underline{B}, \underline{C}, \underline{F}$. Furthermore,

$$
T_{0}=\left[\begin{array}{lll}
I & 0 & 0 \\
0 & I & 0
\end{array}\right], \quad T_{f}=[0,0, I]
$$

where $I$ stands for suitable-order identity matrix and

$$
\pi=\left[\begin{array}{c}
x_{t_{0}}^{2} \\
e_{t_{0}}
\end{array}\right], \quad \chi=k_{t_{f}}^{2}=0 .
$$

Note that, for these specific structural forms,

$$
T_{0}^{\prime} T_{0}+T_{f}^{\prime} T_{f}=I \quad \text { and } T_{f} A T_{0}^{\prime}=0 ;
$$

therefore, conditions (19b) and (19c) are satisfied. Then,

$$
T_{0} A T_{0}^{\prime}=\left[\begin{array}{cc}
A-B^{2} B^{2^{\prime}} S^{2} & 0 \\
0 & A
\end{array}\right],
$$

and $\Phi_{0}(t, \sigma)$ can be split into four submatrices which satisfy

$$
\begin{gathered}
(d / d t)\left[\begin{array}{ll}
\Phi_{0}^{11}(t, \sigma) & \Phi_{0}^{12}(t, \sigma) \\
\Phi_{0}^{21}(t, \sigma) & \Phi_{0}^{22}(t, \sigma)
\end{array}\right]=\left[\begin{array}{cc}
A-B^{2} B^{2^{\prime}} S^{2} & 0 \\
0 & A
\end{array}\right]\left[\begin{array}{ll}
\Phi_{0}^{11}(t, \sigma) & \Phi_{0}^{12}(t, \sigma) \\
\Phi_{0}^{21}(t, \sigma) & \Phi_{0}^{22}(t, \sigma)
\end{array}\right], \\
{\left[\begin{array}{ll}
\Phi_{0}^{11}(\sigma, \sigma) & \Phi_{0}^{12}(\sigma, \sigma) \\
\Phi_{0}^{21}(\sigma, \sigma) & \Phi_{0}^{22}(\sigma, \sigma)
\end{array}\right]=\left[\begin{array}{ll}
I & 0 \\
0 & I
\end{array}\right] .}
\end{gathered}
$$

Therefore,

$$
\begin{aligned}
\Phi^{12}(t, \sigma) & =\Phi^{21}(t, \sigma)=0, & & \\
(d / d t) \Phi_{0}^{11}(t, \sigma) & =\left(A-B^{2} B^{2^{\prime}} S^{2}\right) \Phi_{0}^{11}(t, \sigma), & & \Phi_{0}^{11}(\sigma, \sigma)=I, \\
(d / d t) \Phi_{0}^{22}(t, \sigma) & =A \Phi_{0}^{22}(t, \sigma), & & \Phi_{0}^{22}(\sigma, \sigma)=I .
\end{aligned}
$$

On the other hand,

$$
\begin{aligned}
T_{f} A T_{f}^{\prime} & =-\left(A^{\prime}-S^{2} B^{2} B^{2^{\prime}}\right), \\
(d / d t) \Phi_{f}(t, \sigma) & =-\left(A^{\prime}-S^{2} B^{2} B^{2^{\prime}}\right) \Phi_{f}(t, \sigma), \quad \Phi_{f}(\sigma, \sigma)=I .
\end{aligned}
$$


Thus,

$$
\Phi_{f}(t, \sigma)=\Phi_{0}^{11}(\sigma, t)^{\prime} .
$$

Let us denote $\Phi_{0}^{11}(t, \sigma)$ by $\psi(t, \sigma)$ and $\Phi_{0}^{22}(t, \sigma)$ by $\Phi(t, \sigma)$. Now,

$$
\begin{gathered}
T_{0}^{\prime} \Phi_{0} T_{0}=\left[\begin{array}{ccc}
\psi & 0 & 0 \\
0 & \Phi & 0 \\
0 & 0 & 0
\end{array}\right], \quad T_{f}^{\prime} \Phi_{f} T_{f}=\left[\begin{array}{ccc}
0 & 0 & 0 \\
0 & 0 & 0 \\
0 & 0 & \Phi_{f}
\end{array}\right], \\
T_{0}^{\prime} \Phi_{0}(t, \tau) T_{0} A(\tau) T_{f}^{\prime} \Phi_{f}(\tau, \sigma) T_{f}=\left[\begin{array}{ccc}
0 & 0 & -\Psi(t, \tau) B^{2}(\tau) B^{2}(\tau)^{\prime} \Psi(\sigma, \tau)^{\prime} \\
0 & 0 & 0 \\
0 & 0 & 0
\end{array}\right],
\end{gathered}
$$

and define

$$
F(t, \sigma)=\int_{t_{0}}^{\sigma} d \tau \Psi(t, \tau) B^{2}(\tau) B^{2}(\tau)^{\prime} \Psi(\sigma, \tau)^{\prime}
$$

Then,

Let

$$
\begin{aligned}
P(t, \sigma) & =\left[\begin{array}{ccc}
\Psi(t, \sigma) & 0 & F(t, \sigma) \\
0 & \Phi(t, \sigma) & 0 \\
0 & 0 & 0
\end{array}\right], \\
P^{2}(t, \sigma) & =\left[\begin{array}{ccc}
0 & 0 & -F(\sigma, t)^{\prime} \\
0 & 0 & 0 \\
0 & 0 & \Psi(\sigma, t)^{\prime}
\end{array}\right]
\end{aligned}
$$

$$
\hat{k}_{t}^{21}=E\left[k_{t}^{2} \mid y^{1}\right], \quad \hat{x}_{t}^{21}=E\left[\hat{x}_{t}^{2} \mid y^{1}\right], \quad \hat{e}_{t}^{1}=E\left[e_{t} \mid y^{1}\right] .
$$

Then, the following result follows directly from Lemma 3.1 by identifying $y$ with $y^{1}, z$ with $y^{2}$, and $\gamma$ with $\gamma^{1}$.

Theorem 4.1. The two-person nonzero-sum stochastic differential game with static information, as formulated in Section 2, admits a unique Stackelberg solution with DM1 as the leader. The leader's corresponding Stackelberg strategy $\gamma^{1} \in \Gamma^{1}$ is given by

$$
\begin{aligned}
\gamma^{10}\left(t, y^{1}\right)= & -B^{1}(t)^{\prime}\left[\left(\Psi\left(t_{f}, t\right)^{\prime}-S^{2}(t) F\left(t_{f}, t\right)^{\prime}\right) Q_{1 f} \hat{\hat{x}}_{t_{f}}^{21}+\Phi\left(t_{f}, t\right)^{\prime} Q_{1 f} \hat{e}_{t_{f}}^{1}\right] \\
& -B^{1}(t)^{\prime} \int_{t}^{t_{f}}\left[\left(\Psi(\sigma, t)^{\prime}-S^{2}(t) F(\sigma, t)^{\prime}\right)\left(Q_{1}(\sigma) \hat{\hat{x}}_{\sigma}^{21}+S^{2}(\sigma) \hat{z}_{\sigma}\right)\right. \\
& \left.+\Phi(\sigma, t)^{\prime} Q_{1}(\sigma) \hat{e}_{\sigma}^{1}\right] d \sigma \\
& +B^{1}(t)^{\prime} S^{2}(t) \int_{t_{0}}^{t}\left[F(t, \sigma)\left(Q_{1}(\sigma) \hat{\hat{x}}_{\sigma}^{21}+S^{2}(\sigma) \hat{z}_{\sigma}\right)-\Psi(t, \sigma) \hat{z}_{\sigma}\right] d \sigma,
\end{aligned}
$$


where

$$
\hat{z}_{t}=B^{2}(t) R(t) B^{2}(t)^{\prime}\left[S^{2}(t) \hat{\hat{x}}_{t}^{21}+\hat{k}_{t}^{21}\right],
$$

and $\hat{\hat{x}}_{t}^{21}, \hat{e}_{t}^{1}, \hat{k}_{t}^{21}$ satisfy the differential equations

$$
\begin{aligned}
\dot{\hat{x}}_{t}^{21} & =\left(A-B^{2} B^{2^{\prime}} S^{2}\right) \hat{\hat{x}}_{t}^{21}+B^{1} E\left[E\left[\gamma^{10}\left(t, y^{1}\right)\left|y^{2}\right| y^{1}\right]-B^{2} B^{2^{\prime}} \hat{k}_{t}^{21},\right. \\
\hat{e}_{t}^{1} & =A \hat{e}_{t}^{1}+B^{1}(t) \gamma^{10}\left(t, y^{1}\right)-B^{1}(t) E\left[E\left[\gamma^{10}\left(t, y^{1}\right) \mid y^{2}\right] \mid y^{1}\right], \\
\dot{k}_{t}^{21} & =-\left(A^{\prime}-S^{2} B^{2} B^{2}\right) \hat{k}_{t}^{21}-S^{2} B^{1} E\left[E\left[\gamma^{10}\left(t, y^{1}\right) \mid y^{2}\right] \mid y^{1}\right], \\
\hat{x}_{t_{0}}^{21} & =E\left[E\left[x_{0} \mid y^{2}\right] \mid y^{1}\right], \\
\hat{e}_{t_{0}}^{1} & =E\left[x_{0} \mid y^{1}\right]-E\left[E\left[x_{0} \mid y^{2}\right] \mid y^{1}\right], \\
\hat{k}_{t_{f}}^{21} & =0 .
\end{aligned}
$$

Furthermore, the corresponding unique optimal response strategy of DM2, the follower, is given by

$$
\gamma^{2^{0}}\left(t, y^{2}\right)=-B^{2}(t)^{\prime}\left\{S^{2}(t) \hat{x}_{t}^{2}+k_{t}^{2}\right\},
$$

where the relevant terms are defined by (15) and (16), with $\gamma^{1}$ replaced by $\gamma^{1^{0}}$.

\section{Special Case of Gaussian Statistics}

When the statistics of the random variables involved are jointly Gaussian, some of the expressions that determine the unique Stackelberg strategy of the leader can further be simplified; in particular, it can be shown that the Stackelberg strategy is affine in the available static observation. Toward this end, let us first assume that $x_{0}, y^{1}, y^{2}$ are jointly Gaussian with mean values $\bar{x}_{0}, \bar{y}^{1}, \bar{y}^{2}$, respectively.

Furthermore, let us introduce the appropriate dimensional matrices $\Lambda_{01}, \Lambda_{02}, \Lambda_{12}, \Lambda_{21}$ through the relations

$$
\begin{aligned}
& E\left[x_{0} \mid y^{i}\right]=\bar{x}^{0}+\Lambda_{0 i}\left(y^{i}-\bar{y}^{i}\right), \quad i=1,2, \\
& E\left[y^{i} \mid y^{j}\right]=\bar{y}^{i}+\Lambda_{i j}\left(y^{j}-\bar{y}^{j}\right), \quad i, j=1,2, \quad i \neq j .
\end{aligned}
$$

Now, starting with expressions (14), we note that $\gamma^{10}$ [which satisfies (33)] is also given by

$$
\begin{aligned}
\gamma^{10}\left(\cdot, y^{1}\right)= & -(I+\overline{\mathscr{R}})^{-1}\left\{\tilde{\mathscr{L}}_{1}^{*} \mathscr{Q}_{1} \tilde{r}+\mathscr{L}_{1}^{*} \mathscr{Q}_{2} \mathscr{L}_{2}\left(I+\mathscr{L}_{2}^{*} \mathscr{Q}_{2} \mathscr{L}_{2}\right)^{-1}\right. \\
& \left.\times R\left(I+\mathscr{L}_{2}^{*} \mathscr{Q}_{2} \mathscr{L}_{2}\right)^{-1} \mathscr{L}_{2}^{*} \mathscr{Q}_{2} r\right\}
\end{aligned}
$$


where the relevant terms are defined following (14). It has already been shown in Ref. 12, Appendix 1, in a different context, that $\mathscr{L}_{i}^{*}$ is a linear operator which, when applied on an element of $L_{2 f}\left(\left[t_{0}, t_{f}\right] \times \Omega\right)$ under the Gaussianness assumption, yields an element of $H^{i}$ which is affine in $y^{i}$. A similar analysis readily proves that $\tilde{\mathscr{L}}_{1}^{*}$ also possesses the same property. Then, $r_{t}(\omega)$ being a Gaussian vector for each $t \in\left[t_{0}, t_{f}\right]$, we arrive at the conclusion that, under the Gaussianness assumption, $\gamma^{10}\left(\cdot, y^{1}\right)$, as given by (37), is affine in $y^{1}$. In particular, also in view of (33), it can be written as

$$
\gamma^{1^{0}}\left(t, y^{1}\right)=-B^{1}(t)^{\prime}\left[K(t)\left(y^{1}-\bar{y}^{1}\right)+l(t)\right]
$$

where $K(\cdot)$ is an $\left(n \times m_{1}\right)$-dimensional matrix-valued function and $l(\cdot)$ is an $n$-dimensional vector-valued function, both of them being independent of $y^{1}-\bar{y}^{1}$.

Now, to determine the expressions yielding $K(\cdot)$ and $l(\cdot)$, we substitute the functional form (38) into (33) and also into (34a) through (34d). Firstly, we obtain from (34d)

$$
\hat{k}_{t}^{21}=K^{k}(t) \Lambda_{12} \Lambda_{21}\left(y^{1}-\bar{y}^{1}\right)+l^{k}(t),
$$

where $K^{k}(\cdot)$ and $l^{k}(\cdot)$ uniquely satisfy

$$
\begin{aligned}
\dot{K}^{k} & =-\left(A-B^{2} B^{2^{\prime}} S^{2}\right)^{\prime} K^{k}+S^{2} B^{1} B^{1^{\prime}} K(t), & K^{k}\left(t_{f}\right) & =0, \\
\dot{l}^{k} & =-\left(A-B^{2} B^{2} S^{2}\right)^{\prime} l^{k}+S^{2} B^{1} B^{1} l(t), & l^{k}\left(t_{f}\right) & =0 .
\end{aligned}
$$

Using this solution, together with (38), in (34b), we further obtain

$$
\hat{x}_{t}^{21}=K^{x}(t)\left(y^{1}-\bar{y}^{1}\right)+l^{x}(t),
$$

where $K^{x}(\cdot)$ and $l^{x}(\cdot)$ uniquely satisfy

$$
\begin{array}{r}
\dot{K}^{x}=\left(A-B^{2} B^{2^{\prime}} S^{2}\right) K^{x}-B^{1} B^{1^{\prime}} K(t) \Lambda_{12} \Lambda_{21}-B^{2} B^{2^{\prime}} K^{k}(t) \Lambda_{12} \Lambda_{21}, \\
K^{x}\left(t_{0}\right)=\Lambda_{02} \Lambda_{21}, \\
\dot{l}^{x}=\left(A-B^{2} B^{2^{\prime}} S^{2}\right) l^{x}-B^{1} B^{1^{\prime}} l(t)-B^{2} B^{2^{\prime}} l^{k}(t), \quad l^{x}\left(t_{0}\right)=\bar{x}_{0} .
\end{array}
$$

Thirdly, substitution of the structural form (38) into (34c) yields

$$
\hat{e}_{t}^{1}=K^{e}(t)\left(y^{1}-\bar{y}^{1}\right),
$$

where $K^{e}(\cdot)$ uniquely satisfies the differential equation

$$
\dot{K}^{e}=A K^{e}-B^{1} B^{1} K(t)+B^{1} B^{1^{\prime}} K(t) \Lambda_{12} \Lambda_{21}, \quad K^{e}\left(t_{0}\right)=\Lambda_{01}-\Lambda_{02} \Lambda_{21} .
$$

If we use all these in (33), we finally obtain the following two integral 
equations whose unique solutions yield $K(\cdot)$ and $l(\cdot)$, respectively:

$$
\begin{aligned}
K(t)= & \Psi\left(t_{f}, t\right)^{\prime}-S^{2}(t) F\left(t_{f}, t\right)^{\prime} Q_{1 f} K^{x}\left(t_{f}\right)+\Phi\left(t_{f}, t\right)^{\prime} Q_{1 f} K^{e}\left(t_{f}\right) \\
& +\int_{t}^{t_{f}}\left\{\left[\Psi(\sigma, t)^{\prime}-S^{2}(t) F(\sigma, t)^{\prime}\right]\right. \\
& \times\left[Q_{1}(\sigma) K^{x}(\sigma)-S^{2}(\sigma) B^{2} R B^{2^{\prime}}\left(S^{2}(\sigma) K^{x}(\sigma)\right.\right. \\
& \left.\left.\left.+K^{k}(\sigma) \Lambda_{12} \Lambda_{21}\right)\right]+\Phi(\sigma, t)^{\prime} Q_{1}(\sigma) K^{e}(\sigma)\right\} d \sigma-S^{2}(t) \int_{t_{0}}^{t}\{F(t, \sigma) \\
& \times\left[Q_{1}(\sigma) K^{x}(\sigma)+S^{2}(\sigma) B^{2} R B^{2^{\prime}}\left(S^{2}(\sigma) K^{x}(\sigma)+K^{k}(\sigma) \Lambda_{12} \Lambda_{21}\right)\right] \\
& \left.-\Psi(t, \sigma) B^{2} R B^{2^{\prime}}\left[S^{2}(\sigma) K^{x}(\sigma)+K^{k}(\sigma) \Lambda_{12} \Lambda_{21}\right]\right\} d \sigma, \\
l(t)= & {\left[\Psi\left(t_{f}, t\right)^{\prime}-S^{2}(t) F\left(t_{f}, t\right)^{\prime}\right] Q_{1 f} l^{x}(t) } \\
& +\int_{t_{g}}^{t_{f}}\left\{\left[\Psi(\sigma, t)^{\prime}-S^{2}(t) F(\sigma, t)^{\prime}\right]\right. \\
& \times\left[Q_{1}(\sigma) l^{x}(\sigma)+S^{2}(\sigma) B^{2} R B^{2^{\prime}}\left(S^{2}(\sigma) l^{x}(\sigma)+l^{k}(\sigma)\right]\right\} d \sigma \\
& -S^{2}(t) \int_{t_{0}}^{t}\left\{F ( t , \sigma ) \left[Q_{1}(\sigma) l^{x}(\sigma)+S^{2}(\sigma) B^{2} R B^{2^{\prime}}\left(S^{2}(\sigma) l^{x}(\sigma)\right.\right.\right. \\
& \left.\left.\left.+l^{k}(\sigma)\right)\right]-\Psi(t, \sigma) B^{2} R B^{2^{\prime}}\left[S^{2}(\sigma) l^{x}(\sigma)+l^{k}(\sigma)\right]\right\} d \sigma .
\end{aligned}
$$

The following theorem now summarizes the main result of this section.

Theorem 5.1. When all the random vectors are jointly Gaussian distributed, the two-person nonzero-sum stochastic differential game of Section 2 admits a unique Stackelberg strategy for the leader, which is given by $(38)$, with $K(\cdot)$ and $l(\cdot)$ solved from (42) and (43), respectively, also in view of the relations (39)-(41). The corresponding unique optimal response strategy of the follower is again given by (35).

Proof. As discussed prior to the statement of the theorem, this result follows directly from Theorem 4.1 after verification of the optimal affine structure (38) for $\gamma^{10}$ under the Gaussianness assumption. Substitution of (38) into (33) and an appropriate decomposition readily yields Eqs. (42) and (43).

Remark 5.1. It should be noted that, under the Gaussian statistics, the unique Stackelberg strategy of the leader, as presented in Theorem 5.1, is composed of two parts: (i) a linear transformation on the zero-mean part of the leader's observation, which depends on the statistics of the 
Gaussian random variables involved; and (ii) a linear transformation on the mean value $\left(\bar{x}_{0}\right)$ of the initial state, which is independent of the statistics of the random variables. Hence, we can say that the Stackelberg solution also features a separation property. In fact, the latter part of the Stackelberg strategy of the leader, which involves a linear transformation on $\bar{x}_{0}$, also constitutes a Stackelberg solution to the deterministic version of the nonzero-sum differential game of Section 2, in which the leader has open-loop information and $\bar{x}_{0}$ stands for the initial state vector. In that respect, the solution presented here, i.e.,

$$
\gamma^{10}\left(t, \bar{x}_{0}\right)=-B^{1}(t)^{\prime} l(t)
$$

is less restrictive than the one given in Ref. 3 , since it does not require the linear transformation to be differentiable. Whenever $l(\cdot)$ is differentiable, then the two solutions coincide.

\section{Computational Aspects of the Stackelberg Solution}

In this section, we develop an algorithm to solve numerically for the leader's Stackelberg strategy from Eq. (33) and under general statistics. To this end, let us first observe that the solutions of the differential equations (34b) through (34d) can be obtained through affine transformations on $\gamma^{10}$; and, when these are substituted into (33), together with the linear relation (34a), the right-hand side of (33) becomes an affine function of $\gamma^{1^{\circ}}$. Hence, there exists a linear operator $\mathscr{B}: \Gamma^{1} \rightarrow \Gamma^{1}$ and $b \in \Gamma^{1}$, such that (33) can be written equivalently as

$$
\gamma^{10}\left(t, y^{1}\right)=\left(\mathscr{B} \gamma^{10}\right)\left(t, y^{1}\right)+b\left(t, y^{1}\right) .
$$

Explicit expressions for the operator $\mathscr{B}$ and the function $b$ can readily be obtained in terms of the parameters of the problem.

Now, by an abuse of notation, we can also think of $\mathscr{B}$ as a mapping from $H^{1}$ into $H^{1}$ and of $b$ as an element of $H^{1}$. Then, Eq. (44) can be written equivalently as

$$
u^{1}=\mathscr{B} u^{1}+b
$$

which is known to admit the unique solution

$$
u^{10}=\gamma^{10}\left(t, y^{1}\right) .
$$

Then, a natural algorithm to obtain $u^{10}$ is the following. 


\section{Algorithm}

Step 1. Start with any $u^{1} \in H^{1}$; substitute this into (34a) through (34d) for $\gamma^{10}$, and solve for the corresponding unique $\hat{z}_{t}, \hat{x}_{t}^{21}, \hat{e}_{t}^{1}, \hat{k}_{t}^{21}$.

Step 2. Substitute these into the right-hand side of (33) to generate a new $u^{1} \in H^{1}$.

Step 3. Use the new $u^{1}$ obtained at Step 2 to replace the starting choice at Step 1, and iterate.

Proposition 6.1. Let there exist a $0<\rho<1$ such that

$$
\left\langle u, \mathscr{B}^{*} \mathscr{B} u\right\rangle_{1} \leqslant \rho\langle u, u\rangle_{1}, \quad \forall u \in H^{1} .
$$

Then, the preceding algorithm converges to the unique Stackelberg control $u^{10}$ of the leader, regardless of the initial choice.

Proof. The result readily follows, since $H^{1}$ is a Hilbert space and $\mathscr{B}$ is a linear contraction operator under (46).

If the condition of Proposition 6.1 is not satisfied, then the proposed algorithm in general will not converge. However, in such a situation, it is still possible to develop a convergent algorithm by starting, this time, with Eq. (37), which we rewrite as

where

$$
u^{1}=-\overline{\mathscr{R}} u^{1}+\tilde{\tilde{r}}
$$

$$
\tilde{r} \stackrel{\Delta}{=} \tilde{\mathscr{L}}_{1}^{*} \mathscr{Q}_{1} \tilde{r}+\mathscr{L}_{1}^{*} \mathscr{Q}_{2} \mathscr{L}_{2}\left(I+\mathscr{L}_{2}^{*} \mathscr{Q}_{2} \mathscr{L}_{2}\right)^{-1} R\left(I+\mathscr{L}_{2}^{*} \mathscr{Q}_{2} \mathscr{L}_{2}\right)^{-1} \mathscr{L}_{2}^{*} \mathscr{Q}_{2} r
$$

Now, if the operator norm of $\bar{R}$ is less than unity, i.e.,

$$
\|\overline{\mathscr{R}}\|_{0}<1 \text {, }
$$

we have the algorithm

$$
u_{j+1}^{1}=-\overline{\mathscr{R}} u_{i}^{1}+\tilde{r}
$$

which is directly obtainable from (47a) and is convergent, since $\overline{\mathscr{R}}$ is a linear contraction operator and $H^{1}$ is a Hilbert space. If the condition

$$
\|\overline{\mathscr{R}}\|_{0}<1
$$

is not satisfied, then we add $\theta I u^{1}$ to both sides of $(47 \mathrm{a})$ to obtain

$$
(1+\theta) I u^{1}=(\theta I-\overline{\mathscr{R}}) u^{1}+\tilde{\tilde{r}},
$$

where $I: H^{1} \rightarrow H^{1}$ is the identity operator and $\theta$ is a scalar satisfying

$$
2 \theta \geqslant\|\overline{\mathscr{R}}\|_{0} \text {. }
$$


Then, the algorithm

$$
u_{j+1}^{1}=[1 /(1+\theta)](\theta I-\overline{\mathscr{R}}) u_{j}^{1}+[1 /(1+\theta)] \tilde{r}
$$

converges to the unique solution $u^{10} \in H^{1}$, a result which follows from a convergence proof given in Ref. 13 for a similar algorithm, since $\overline{\mathscr{R}}$ is a nonnegative self-adjoint operator. It should be noted, however, that this algorithm is computationally more difficult than the first one proposed in this section, since it involves the inversion of an integral operator in determining $\tilde{\tilde{r}}$ (though, this inversion has to be done only once, and not at every iteration point).

\section{Conclusions}

In this paper we have obtained the Stackelberg solution of a class of stochastic linear-quadratic differential game problems in which both players have access to static information concerning the initial state of the game. Existence and uniqueness of the solution is established, and the leader's optimal strategy is shown to satisfy a particular integral equation. For the special case when all the random variables have jointly Gaussian statistics, this integral equation admits a solution which is affine in the observation of the leader. We have also discussed in the paper numerical aspects of the Stackelberg solution under general statistics, and have developed algorithms which converge to the unique Stackelberg control of the leader. As a by-product of our analysis, we have also obtained the solution of a nonstandard stochastic control problem, which is formulated and solved in Section 3 in more general terms than required in the solution of the Stackelberg problem.

One natural extension of the results of this paper would be to obtain the feedback Stackelberg solution of linear-quadratic-Gaussian continuoustime differential games in which the players make sampled observations of the state and share this information with a one-step delay (as formulated in Ref. 12 within a team framework). In other words, a counterpart of the results of Ref. 10 in continuous-time can be obtained by utilizing the theory developed in this paper under static information. A repeated application of Theorem 5.1 would lead to the conclusion that the feedback Stackelberg strategies in such a differential game will again be affine in the information available to the players; derivation of these strategies, however, requires a rather involved analysis, details of which will be presented in a future paper. 


\section{References}

1. VON STACKELBERG, H., The Theory of Market Economy, Oxford University Press, Oxford, England, 1952.

2. CHEN, C. I., and CRUZ, J. B., JR., Stackelberg Solution for Two-Person Games with Biased Information Patterns, IEEE Transactions on Automatic Control, Vol. AC-17, No. 5, 1972.

3. SIMAAN, M., and CRUZ, J. B., JR., On the Stackelberg Strategy in Nonzero-sum Games, Journal of Optimization Theory and Applications, Vol. 11, No. 5, 1973.

4. SIMAAN, M. and CRUZ, J. B., JR., Additional Aspect of the Stackelberg Strategy in Nonzero-Sum Games, Journal of Optimization Theory and Applications, Vol. 11 , No. 6, 1973.

5. BASAR, T., Information Structures and Equilibria in Dynamic Games, New Trends in Dynamic Systems Theory and Economics, Edited by M. Aoki and A. Marzollo, Academic Press, New York, New York, 1979.

6. CRUz, J. B., JR., Leader-Follower Strategies For Multilevel Systems, IEEE Transactions on Automatic Control, Vol. AC-23, No. 2, 1978.

7. BASAR, T., and SElbUZ, H., Closed-Loop Stackelberg Strategies with Applictions in the Optimal Control of Multilevel Systems, IEEE Transactions on Automatic Control, Vol. AC-24, No. 2, 1979.

8. Castanon, D., and Athans, M., On the Stochastic Dynamic Stackelberg Strategies, Automatica, Vol. 12, No. 2, 1976.

9. BAŞAR, T., Hierarchical Decision Making under Uncertainty, Dynamic Optimization and Mathematical Economics, Edited by P. T. Liu, Plenum Press, New York, New York, 1979.

10. BAŞAR, T., Stochastic Stagewise Stackelberg Strategies For Linear Quadratic Systems, Stochastic Control Theory and Stochastic Differential Systems, Edited by M. Kohlmann and W. Vogel, Springer-Verlag, Berlin, Germany, 1979.

11. Fleming, W. H., and Nisio, M., On the Existence of Optimal Stochastic Controls, Journal of Mathematics and Mechanics, Vol. 15, pp. 777-794, 1966.

12. BAGHI, A., and BAŞAR, T., Team Decision Theory for Linear Continuous-Time Systems, Twente University of Technology, Enschede, Holland, Department of Applied Mathematics, Memorandum No. 274, 1979.

13. Allwright, H. C., Contraction Mapping Algorithm with Guaranteed Convergence, Proceedings of the 5th IFAC Congress, Paris, France, 1972. 